\title{
A Self Driving Car using Machine Learning and IOT: A Review
}

\author{
${ }^{1}$ Prof.Omprakash Yadav, ${ }^{2}$ Aman Sharma, ${ }^{2}$ Dion Philip, ${ }^{2}$ Boris Alexander \\ ${ }^{1}$ Professor, ${ }^{2}$ Student, Department of Computer Engineering, Xavier Institute of Engineering, Mumbai, India \\ Opposite S.L.Raheja Hospital, Mahim Causeway, Mahim (West), Mumbai, Maharashtra 400016, India \\ amansharma44007@gmail.com,Omprakash.y@xavier.ac.in,dionphilip28@gmail.com,borisalexander
}

\begin{abstract}
In the fashionable era, the vehicles ar centered to beautomated to grant human driver relaxed driving. within the within the numerous aspects are thought of that makesa vehicle machine-driven. Google, the most important network has startedworking on the self-driving cars since 2010 and still developingnew changes to grant an entire new level to the automatedvehicles. during this paper we've got centered on 2 applications of anautomated automobile, one during which 2 vehicles have same destinationand one is aware of the route, wherever different don't. The followingvehicle can follow the target (i.e. Front) vehicle mechanically.The other application is machine-driven driving throughout the heavytraffic jam, thence restful driver from endlessly pushingbrake, accelerator or clutch. the concept delineated during this paperhas been taken from the Google automobile, defining the one side hereinafter thought is creating the destination dynamic. This canbe done by a vehicle mechanically following the destination ofanother vehicle. Since taking intelligent choices within the within the a difficulty for the machine-driven vehicle therefore this side has beenalso into consideration during this paper. Key Words-Automated driving throughout rush hours, dynamic, destination, self-driving, OpenCv, A.I.
\end{abstract}

\section{I.Introduction}

Automated vehicles square measure technological development in thefield of cars. though the machine-driven vehicles square measure forease of man however they're the foremost highpriced vehicles.In the paper considering the various options and also the price, ona tiny scale a 3 wheel conveyance Robotic epitome hasbeen designed which will mechanically reach the destination ofanother vehicle to that it's alleged to follow.We have centered on 2 applications of AN AutomatedVehicles here and designed a epitome vehicle for that.The one major issue is throughout significant significant a driver hasto endlessly push brake, accelerator and clutch to moveto destination slowly. we've got projected an answer to relax the driving force therein state of affairs by creating vehicle good enoughto build choices mechanically and move by maintaining aspecified distance from vehicles and obstacles around.The second issue is once 2 vehicles have the samedestination however one among the drivers doesn't recognize its route. Thedriver will build his vehicle follow the front vehicle if they areknown and share their location to succeed in identical destination.A three-wheel Mobile automaton is employed for analysis is given.The Mobile automaton consists of multiple sensors, that helps itto communicate with Google Maps API (Application ProgramInterface) and makes it confirm obstacles so as to followthe route and move swimmingly. The Mobile automaton connectsdirectly to Google Maps API exploitation
GPRS Module, gets routeand moves therein direction. whereas the supersonic sensors, which are used for epitome style, helps to avoidobstacles on run time.The traffic state of affairs in India ends up in style this projectprototype, that aims at reposeful driver and making anautomated vehicle whose destination is dynamic in contrast to Googlecar, whose destination is static and fixed. This analysis hasbeen a requirement for Pakistan if enforced in real time.The remaining paper is organized because the Section II showsthe connected work worn out worn out. Later we've got discussedour methodology to implement this idea and solve the problem inSection III. Then we've got shown performance analysis of ourMobile automaton in Section IV and finally, we've got concludedthe paper in Section V.

\section{Literature}

After the event of the autopilot airplanes [1], self-driven sailboats and ships; the misleadingly modest dream thathas seldom ventured on the far side the pages of science fiction since our grandparent's youth is that the selfdriving automobile [2]. By thepassage of your time, a lot of work has been allotted within the areato create cars self-driven [3], [4] however because of technologicaladvancement within the roads and also the increasing population hasmade difficult for this dream to changing into true.In the pre-computer days of the Nineteen Thirties, the driverless carswere solely the science fiction things. however the event ofthe computing machine created doable to dream of self-drivenvehicles outside the fiction. By the Nineteen Sixties the selfdriven carshave been unreal to navigate on normal streets 
on theirown. German pioneer Ernst Dickmanns, within the Nineteen Eighties, got aMercedes van to drive many miles autonomously onhighways, an amazing exploit particularly with the computingpower of the time [2]. within the mid2000s, the Defense AdvancedResearch comes Agency (DARPA) sorted out the GrandChallenges wherever teams assembled to traumatize self-driving vehicles. In 2009, Google began the self-driving carventure, as well as colleagues United Nations agency had effectively devotedyears to the innovation. By 2012 the Google automobile hits theroad for testing. By the passing years, the automobile is developedand equipped with multiple sensors, radars, lasers, GlobalPositioning System (GPS), it uses heavily elaborated maps, andmany different things to soundly drive and navigate itself with nohuman interaction. The automobile can't solely drive itself however itcan be lay on its own, it will maintain freeways, Camerasare wont to wont to find objects that square measure then processedby the pc at intervals the automobile [5]. In could 2014, Googlepresented a brand new construct for his or her driverless automobile that had neithera handwheel nor pedals and undraped a totally functioningprototype in December of that year that they planned to check in2015[6]. In summer 2015, Google launched and tested somedifferent options wherever every prototypes speed is capped ata neighborhood-friendly $25 \mathrm{mph}$, and through this part safetydrivers aboard with a removable handwheel, acceleratorpedal, and treadle that permit them to require over drivingif required [7]. when several productive roads testing of Googlecar has created to believe some years roads are going to be safelyoccupied with selfdriven cars. The authors in [8], [9], [10] have developed unmannedvehicle prototypes during which they need worked on the obstacleavoidance and path coming up with [11].In this paper, we've designed 2 applications of anautonomous vehicle, which might facilitate the driving force to relax forthe sure length of your time. This paper presents a constructin that the modified concept of Google automobile is concentrated, theGoogle automobile should reach the static destination automatically; in our image, we've created the destination dynamic.Here our destination is additionally a vehicle that is moving on acertain route. Our image can follow that vehicle. Anotherapplication that we've enforced here was to tackle heavy traffic congestion and permit the vehicle to maneuver automaticallyduring that traffic congestion.

\section{Implementation Methodologies}

Our example model shows some work on each the appli-cation that we've mentioned during this paper. Fig.1 shows the diagram of example Mobile mechanism (Vehicle).

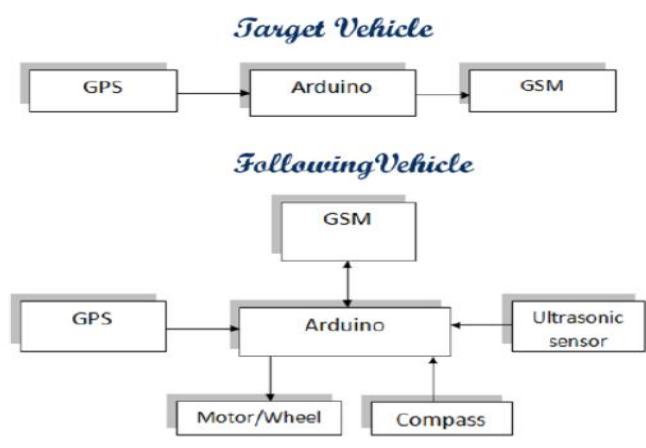

Fig 1: Block Diagram

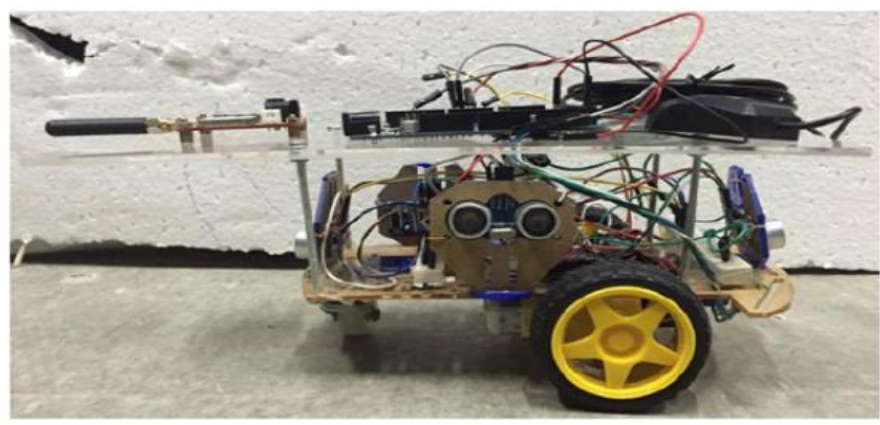

Fig 2: Design of Self Driving Car

Our main focus was on Following Vehicle, that detectsand avoids obstacles, coordinate with Google Maps API, getroute and follow the route. for one more application, it checksvehicles around and mechanically moves slowly behind thetraffic till it gets out of traffic jam state of affairs. The functionof the Target vehicle is simply to produce the coordinates toFollowing Vehicle, that also are not static because the Targetvehicle is moving towards its destination. Fig.2 shows one application within which it follows anothervehicle.Fig. 3 shows another application within which vehicle automati-cally follow the front vehicle and maintains specified distancefrom vehicles around. The vehicle mechanically moves andhence relaxes the driving force.The Fig. a pair of and three shows the hardware implementation.

Thiswhole project involves the 2 the 2 the 2 theTarget Vehicle and second as Following vehicle. The Targetvehicle fetches its existing location coordinates through GPS and sends to Arduino then these coordinates within the kind ofthe message has been sent to Following vehicle once everycertain time of interval.

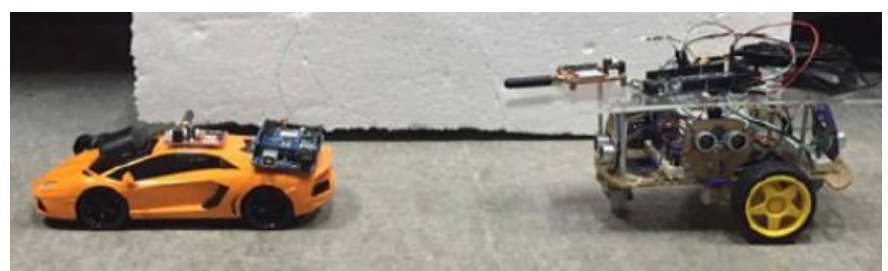

Fig 3: Scenario How We Tested It. 
The Following vehicle whenever receives a message throughGSM, the message is shipped to Arduino. Arduino then decodesthe message and fetches the coordinates of Target vehicle.Since Arduino already has its own coordinates (at FollowingVehicle side). Through GPRS arduino then connects to theGoogle Maps and compares the prevailing location coordinatesof Target vehicle received through the message with existinglocation coordinates of the subsequent vehicle; so tries tofind the littlest doable route to succeed in the Target vehicle.Arduino in spite of everything the process gets the direction from GoogleAPI. This direction is then compared with Robot's currentdirection and once rotating vehicle in this direction, the vehiclestarts moving forward. so vehicle can continue attractive thecurrent location received from the Target vehicle once everycertain time span and can continue move in this direction andthis method continues till the vehicle reaches the destination vehicle's location.
The Following vehicle within the middle of following the Targetvehicle can stick with it trying to find the obstacle which may return. To look for the obstacle, unhearable sensors are used.Ultrasonic Sensors are are round the vehicle sothat the vehicle find the obstacle and find removed from it. Forinstance if there's a wall or a pedestrian before of the vehicle, the unhearable sensors at the front aspect of the vehicle can detectand the vehicle can communicate the safe aspect, another possiblescenario may are available that there ar 3 obstacles; infront, at the proper aspect and at the left aspect of the vehicle,the unhearable sensors can once more find the obstacles associated thevehicle can then moves backward albeit there's an obstaclebehind, it'll stop for a flash and so once more appearance for theobstacles from the start so deciding the route on thebasis of circumstances.

The complete the whole of Following vehicle is shown inFig.4.

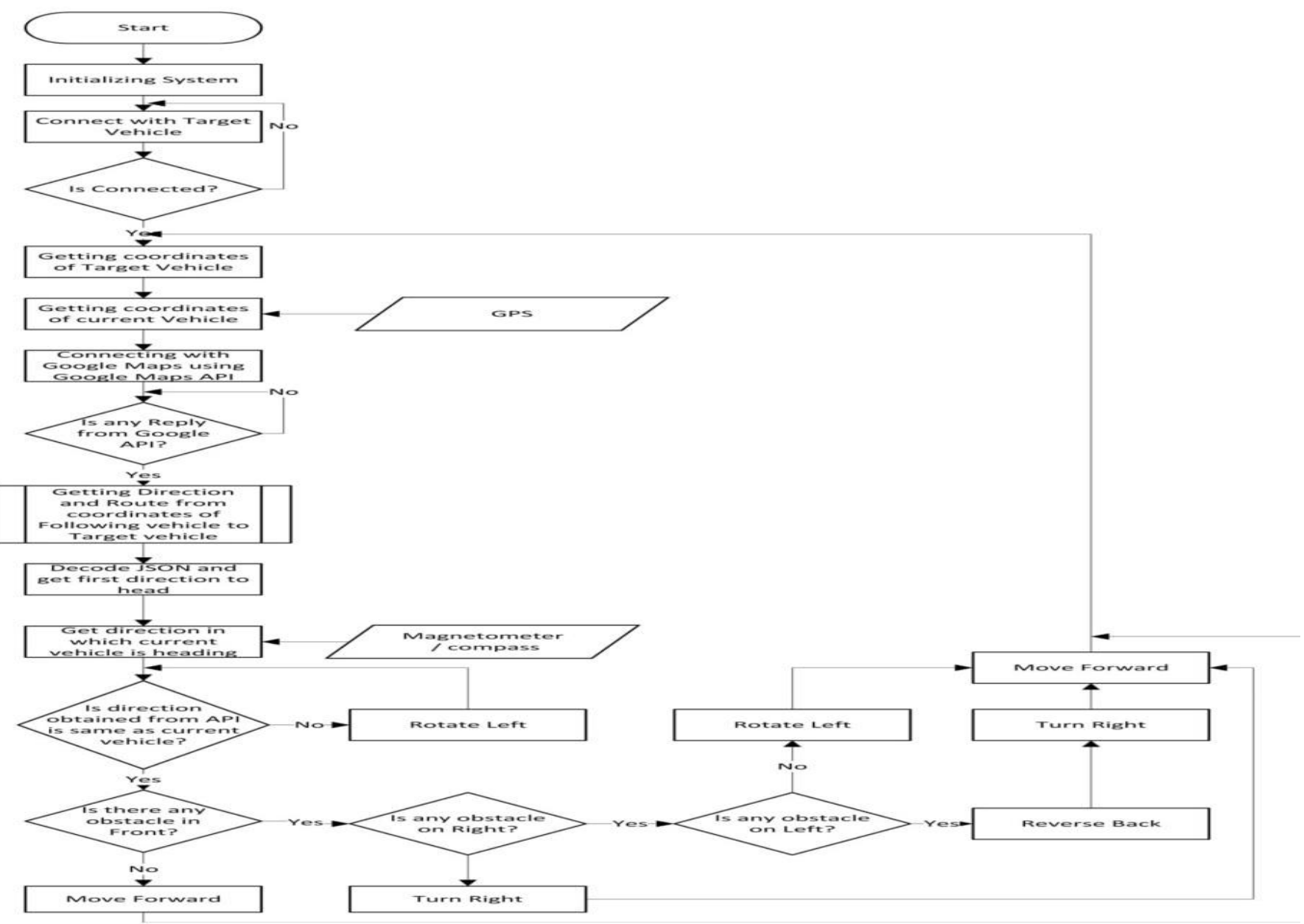

Fig 4. Flowchart of design robot 


\section{Results}

The project has been tested at Mehran University ofEngineering and Technology, Jamshoro and located workingsmoothly as shown in Figure five, 6 and 7. The front vehicleis moving on its thanks to some destination, whereas the followingvehicle (at back) is obtaining GPS location of the front vehicleand moving towards it by obtaining directions and instructionsfrom Google Maps exploitation Google Maps API. Blue line route shows the route taken by the Front vehicle whereas the Redcolored line shows the route taken by the subsequent vehicleafter obtaining directions from Google Maps.

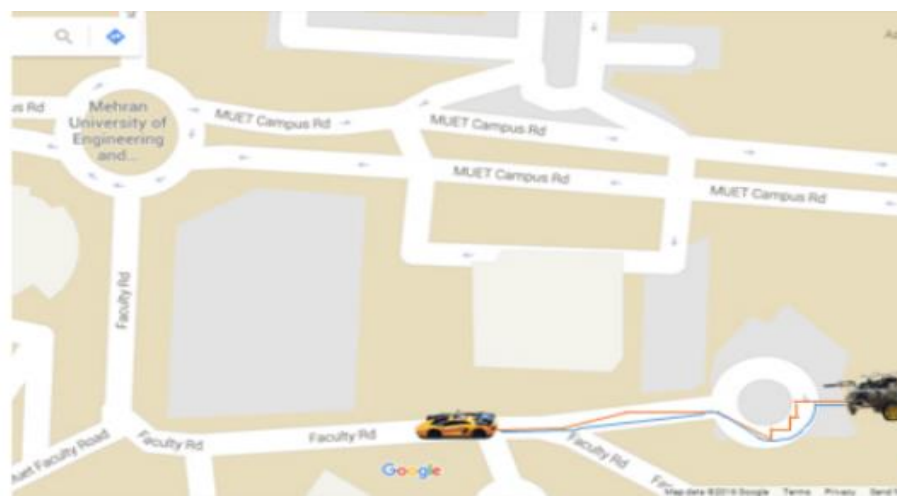

Fig 5: Experimenting Self Driving Mechanism

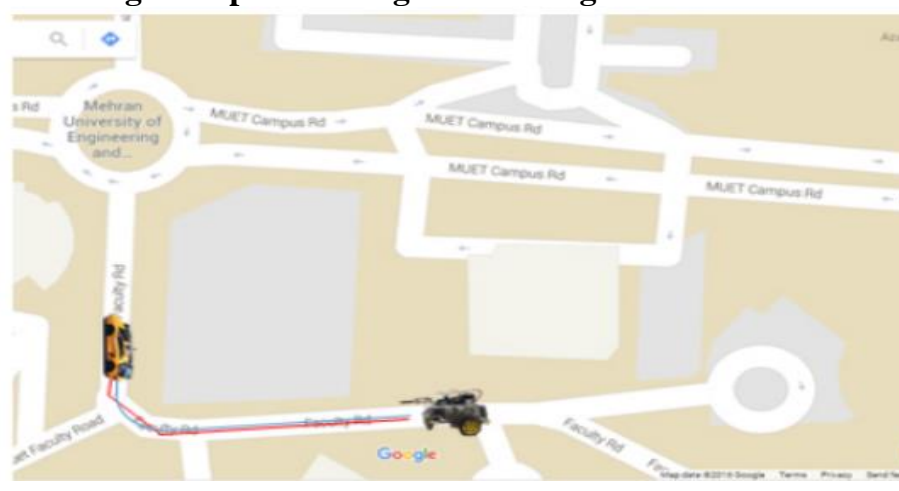

Fig 6: Experimenting During Turns.

By testing the vehicle in real time, we've got conjointly observedthat though the target vehicle takes the incorrect route, thefollowing vehicle can follow the correct route as a result of it isconnected to Google Maps. because it could be a image and vehicleis terribly tiny, therefore it's terribly slow however if the system is implemented in real vehicles then this might facilitate in solving the problem in correct way as mentioned problems in real time.

\section{Conclusion}

This is a sophisticated step for autonomous driving vehicles. With the assistance of this formula, vehicles is set to auto-matically navigate to the destination location by continuouslyreceiving the direction from another vehicle moving ahead tothe same destination.The robotic vehicle routes itself with the steerage of an-other vehicle moving ahead to constant destination, therefore, deviations in time will occur. The goal of navigation processfor a mechanismic vehicle is to maneuver the robot to a familiar desti-nation in Associate in Nursing unknown surroundings. The navigation planningis one amongst the very important aspects of autonomous systems. once therobotic vehicle really starts to maneuver towards the planned route it should it should obstacles from the present locationto the destined location, thus the robotic vehicle should avoidthe obstacles Associate in Nursingd follow an best route to succeed in the destinedposition. The potential applications of this robotic vehicle areto use these styles of autonomous vehicle on highways or heavytraffic roads. These styles of autonomous vehicles may also beused once a driver travels to the new areas. it's Associate in Nursing improvednavigation system for autonomous vehicles.

\section{References}

[1] M. Frutiger and C. Kim, "Digital Autopilot Test Platform with Reference Design and Implementation of a 2-Axis Autopilot for Small Airplanes," Departament of Electrical and, pp. 1-24, 2003.

[2] M. Weber, "Where to? A History of Autonomous Vehicles," 2014. [Online].

[3] D. Helbing, "Traffic and related self-driven many-particle systems," Reviews of Modern Physics, vol. 73, no. 4, pp. 1067-1141, dec 2001.

[4] P. Rau, "Target Crash Population of Automated Vehicles," in In 24th International Technical Conference on the Enhanced Safety of Vehicles (ESV), 2015, pp.

[5] P. Jaroszek and M. Trojnacki, "Localization of the Wheeled Mobile Robot Based on Multi-Sensor Data Fusion," Journal of Automation, Mobile Robotics \&Intelligentcc Systems, vol. 9, no. 3, pp 